ISSN 2409-2665

Journal of Logistics, Informatics and Service Science

Vol. 8 (2021) No. 2, pp. 103-118

DOI:10.33168/LISS.2021.0206

\title{
Evaluation of Distance Learning Resilience During COVID-19 Pandemic using ANFIS
}

\author{
Nazanin Pilevari ${ }^{1}$, Saeideh Memarian ${ }^{2}$, Mohammad Shokouhifar ${ }^{3}$ \\ ${ }^{1}$ Department of Industrial Management, West Tehran Branch, Islamic Azad \\ University, Tehran, Iran \\ ${ }^{2}$ Department of Computer Engineering, Science and Research Branch, Islamic Azad \\ University, Tehran, Iran \\ ${ }^{3}$ Department of Electrical and Computer Engineering, Shahid Beheshti University, \\ Tehran, Iran \\ + m_shokouhifar@sbu.ac.ir
}

\begin{abstract}
Given the growing usage of e-learning systems during COVID-19 epidemic and expansion of internet-based infrastructure, a resilient approach for e-learning systems is highly required. This paper proposes an Adaptive NeuroFuzzy Inference System (ANFIS) to evaluate e-learning resilience. In the ANFIS model, five substantial factors including individual, technology, content, agility, and assessment/support factors are considered as fuzzy inputs, while e-learning resilience is considered as a single output. The proposed ANFIS model has been successfully implemented for e-learning resilience measurement during COVID19 epidemic in virtual Iranian university. Statistical analysis demonstrated that there was no meaningful difference between experts' opinions and our proposed procedure for e-learning resilience measurement. Sensitivity analysis via the proposed model on changing the different factors showed significant sensitivity to changes in the agility factor. The proposed model can be used in all educational institutions to evaluate the improvement of resilience in e-learning systems. To implement the model for an organization, the values of the designed ANFIS model should be defined specifically for the organization and the corresponding model need to be simulated by examining the involved components and relationships.
\end{abstract}

Keywords: Education systems, distance learning, resilience, COVID-19, ANFIS 


\section{Introduction}

COVID-19 is not only a major health crisis; it is changing the structure of global order in business and economy. From March 11, 2020, most activities around the world were suspended following World Health Organization (WHO) declaration of Covid-19 as an epidemic (Al-Balas et al., 2020). It has caused new health challenges in different sectors such as architectural design (Alhusban et al., 2021), construction sector (Simpeh \& Amoah, 2021), blood supply chain (Shokouhifar et al., 2021), and education systems (Al-Balas et al., 2020; Dignan, 2020). Without using internet-based technologies such as e-learning, standard health protocols during COVID-19 (e.g., social distancing, quarantine, lockdown, health consultation, and social awareness) would be ineffective which also damages to economic, social, and educational sectors (McKenzie, 2020). Therefore, improvement of resilience can be one factor in mitigating the unfavorable effects and severity of disorders and disturbances caused by the outbreak of COVID-19.

By outbreaking COVID-19, face-to-face education systems have shifted their focus to e-learning systems after a short hiatus. Modernizing education systems by Information and Communication Technology (ICT) has become an interesting topic, which is mainly implemented as e-learning systems. By embracing such technologies as digital video conference programs such as Google Classroom, Microsoft platform, Zoom, and Webex, education industries attempt to enhance elearning systems globally (Dignan, 2020). E-learning is a learning/teaching platform that depends on electronic devices and technology.

Resilience is the ability of a system to decrease the likelihood of a shock, absorb it in the event of a sudden occurrence (sudden decrease in performance), and recover rapidly in consequence (returning to its normal performance) (Francis et al., 2014). In addition to functionality and optimal conditions for network development, many studies have investigated collaborative networks (Shahabi et al., 2019; Bonomi et al., 2020). However, an insignificant body of research has been dedicated to network flexibility and resilience under social and economic crises. Although different perceptions in this field have been proposed and discussed, the concept of resilience is open to debate and varying interpretations in different scientific fields.

Potential attributes of Adaptive Neuro-Fuzzy Inference System (ANFIS) such as considering fuzzy membership functions for resilience capabilities, training these functions via an adaptive procedure, and using fuzzy concepts to handle uncertainties, have motivated us to develop a fuzzy knowledge-based model based on ANFIS for the evaluation of e-learning resilience during COVID-19 epidemic. The key contributions of the paper can be described as follows:

A fuzzy knowledge-based model based on ANFIS is presented as an efficient model to evaluate the resilience of distance learning systems. 
- In the proposed ANFIS model, the e-learning resilience is measured based on 22 features from five factors including individual, technology, content, agility, and assessment/support factors.

- The objective function of ANFIS is to reduce the Root Mean Square Error (RMSE) between predicted resilience and actual resilience achieved by experts.

- Sensitivity analysis is done to find the impact of changes in different factors of ANFIS to the resilience of e-learning during COVID-19 epidemic.

- The proposed model has been successfully implemented to evaluate elearning resilience in a real case study on virtual Iranian university during the outbreak of COVID-19.

In remainder of the paper, the existing methods in literature are reviewed in Section 2. Section 3 proposes the ANFIS model to evaluate e-learning resilience. Section 4 is devoted to implement the ANFIS model in line with the case study of virtual Iranian university, and discuss sensitivity analysis and findings. Section 5 concludes the paper with some future works.

\section{Literature Review}

\subsection{E-learning during COVID-19 pandemic}

With rapid growth of multimedia systems, networking technologies, and internetbased learning, traditional learning is shifting to virtual learning environments (Moreira et al., 2018). Expansion of new learning methods in educational systems and subsequently, the flexibility of the learners and teachers were improved by the development of Information and Communications Technology (ICT) in education (Naqshbandi et al., 2017). The possibility of distance learning and knowledge sharing through the internet can be facilitated by e-learning with hardware infrastructure and software programs. E-learning is one of the most common technological tools to improve the education process, which can be expressed as the delivery of knowledge through internet (Aljawarneh 2020).

In many countries, educational institutions have been suspended during the COVID-19 outbreak. According to UNESCO (2020), it has caused several disruptions to education systems, leading dropout of school in about 1.6 billion learners in 190 countries. It also threatens the future of a generation with 24 million learners at the risk of dropout. Without the use of ICT and e-learning in education systems, there would be harmful consequences in terms of education and society. Almulhim et al. (2020) have evaluated the effect of e-learning systems on medical students and their outlook during COVID-19 epidemic. Addressing how internet has proved to be robust in successfully coping with these challenges during maintenance of the university operations, Favale et al. (2020) evaluated the unexpected changes observed in campus traffic during COVID-19 epidemic. 
Drawing on findings by Shingal (2020), salient features of the post-COVID-19 education models, areas to be considered, and various e-learning techniques, can be listed in Table 1.

\subsection{E-learning resilience assessment}

Academic leaders need to be conscious of the national and international rules and politics influencing the organization of stakeholders' perspectives, and therefore, being resilient against pressures, flexible to changes, and creative in making opportunities in critical and risky situations. By testing the resilience of the model for different users, De Meo et al. (2017) evaluated the e-learning resilience for the users in a social network. Sabatino (2019) examined the relation of the resilience and social capital, and demonstrated that although the presence of social capital elements is important, it is not important for identifying the economic and social resilience.

Describing the processes of promoting resilience through e-learning, AyebiArthur (2017) organized the dominant themes extracted from data analysis and discussed about crisis, ICT, e-learning technologies, and strategic planning of the elearning systems. Having designed the foundation of resilient programs for addressing lack of professional support for meeting the needs of children influenced by online courses and video-based coaching, Lipscomb et al. (2019) studied the resilience considering the feasibility of learning and practices consistent with the trauma-responsive care. For a more detailed study, readers are recommended to refer to Van de Laar (2020).

Table 1: Salient features of the post-COVID-19 education model

\begin{tabular}{|c|c|c|}
\hline Salient Features & Focus & $\begin{array}{c}\text { Various Techniques for E- } \\
\text { learning }\end{array}$ \\
\hline - Post-COVID-19 & - Developing experiential & - Distance learning \\
mobile & learning material for & - Correspondence learning \\
learning/distance & online classes. & - Learning from home \\
learning will flourish & - Learning/teaching cases & - Massive online open courses \\
- Experiential learning & for case studies. & - Learning through interactive TV \\
will become more & - Simulation games to & channels \\
widespread & explain concepts. & - Video apps such as Youtube, \\
- Social media will & - Role-playing & Vimeo, etc. \\
emerge as important & demonstrating models. & - Learning through apps such as \\
platforms for & - Story-based examples, & Byjus, Unacademy, Udemy, \\
information exchange & questionnaire, and group & Lynda, etc. \\
among teachers, & discussions. & - Learning through e-content blogs, \\
educators, parents, & - Development of & articles, and pdf notes \\
students, \& education & interactive online content & - Self learning through e-books and \\
institutes & to keep students engaged. & other digital content \\
\hline
\end{tabular}




\begin{tabular}{|c|c|c|}
\hline - More emphasis on the & - Development of a & - Listening audiobooks \\
study through & standard common & - Live meeting apps such as \\
simulation tools, case & interface for all users & Google Classroom, Google Meet, \\
studies, stories, and & which is safe, secure, and & Zoom, Cisco Webex, Microsoft \\
examples will be done. & convinient. & team, etc. \\
& & \\
\hline
\end{tabular}

\section{Proposed ANFIS Model for E-learning Resilience Assessment}

Fuzzy Inference System (FIS) is a knowledge-based reasoning model which utilizes fuzzy logic to model uncertainties of the system (Shokouhifar, 2021a). There are different FISs in the literature, e.g., Mamdani and Takagi-Sugeno. The Mamdani FIS considers both input and output to be expressed using fuzzy relational equations in rule-based forms (Shokouhifar, 2021b), while the Takagi-Sugeno FIS formulates the outputs of each fuzzy rule through crisp functions rather than fuzzy equations (Shokouhifar and Jalali, 2017). ANFIS (jang, 1993) is a combination of fuzzy logic and Artificial Neural Networks (ANNs). It utilizes a supervised learning model to learn the relation between inputs and outputs based on the Takagi-Sugeno FIS (Pilevari et al., 2021).

In this paper, three methods of documentation, fuzzy Delphi method (Nabizadeh et al., 2021), and survey were applied to collect the required information for establishing the theoretical foundations of research and forming a conceptual model. To achieve this purpose, the documentary and library method as well as the latest scientific articles and books related to the resilience of e-learning were used. Also, for refining and finalizing the components of resilience to design the proposed conceptual model, fuzzy Delphi method was applied in line with the consensus of experts. The overall flowchart of designing the proposed ANFIS-based resilience model of the e-learning services can be shown in Fig. 1. 


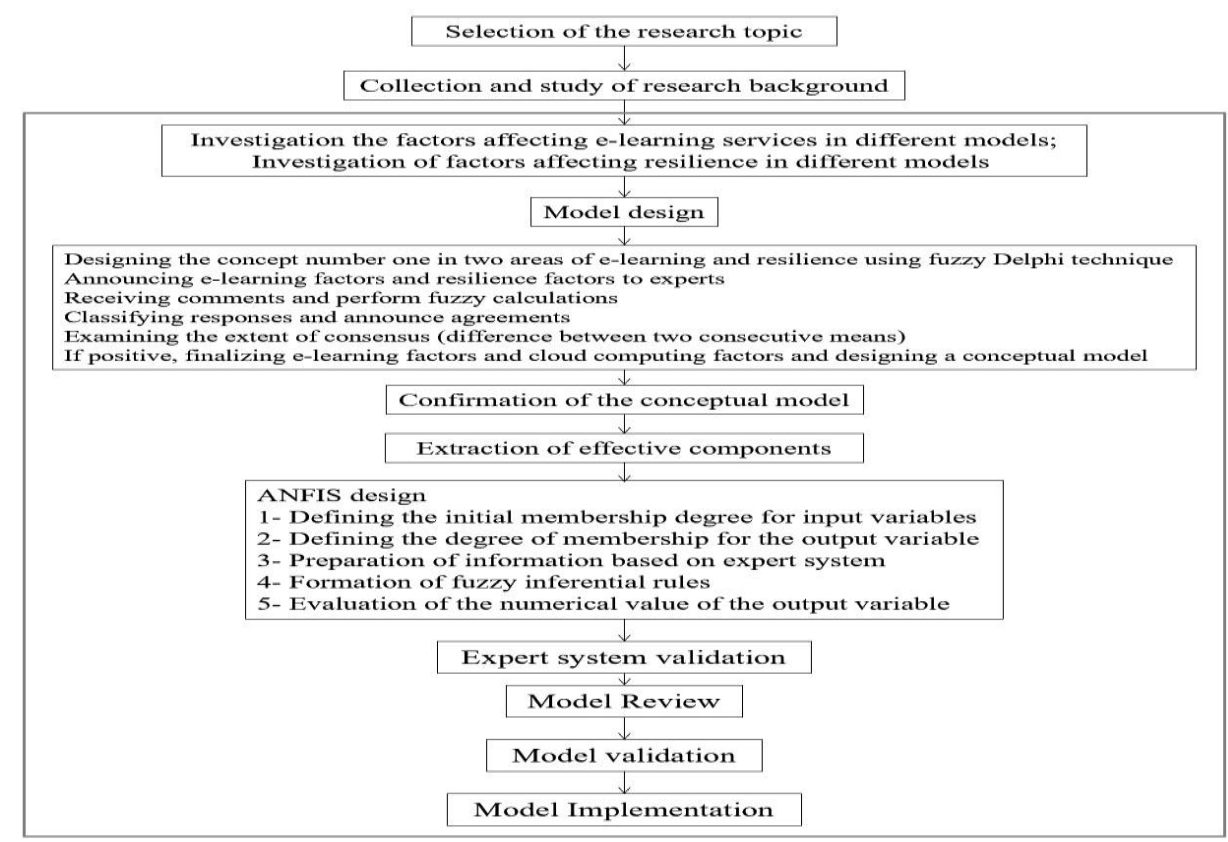

Fig. 1: E-learning resilience evaluation model based on ANFIS.

\subsection{Resilience factors}

The basis for designing a resilience model of e-learning systems was determined upon identifying the main indicators of resilience enablers, the relevant subindicators based on the existing literature, and the knowledge of experts (decision group). As seen in Fig. 2, totally 22 features in five categories including individual, assessment and support, content, agility, and technology factors were obtained. 


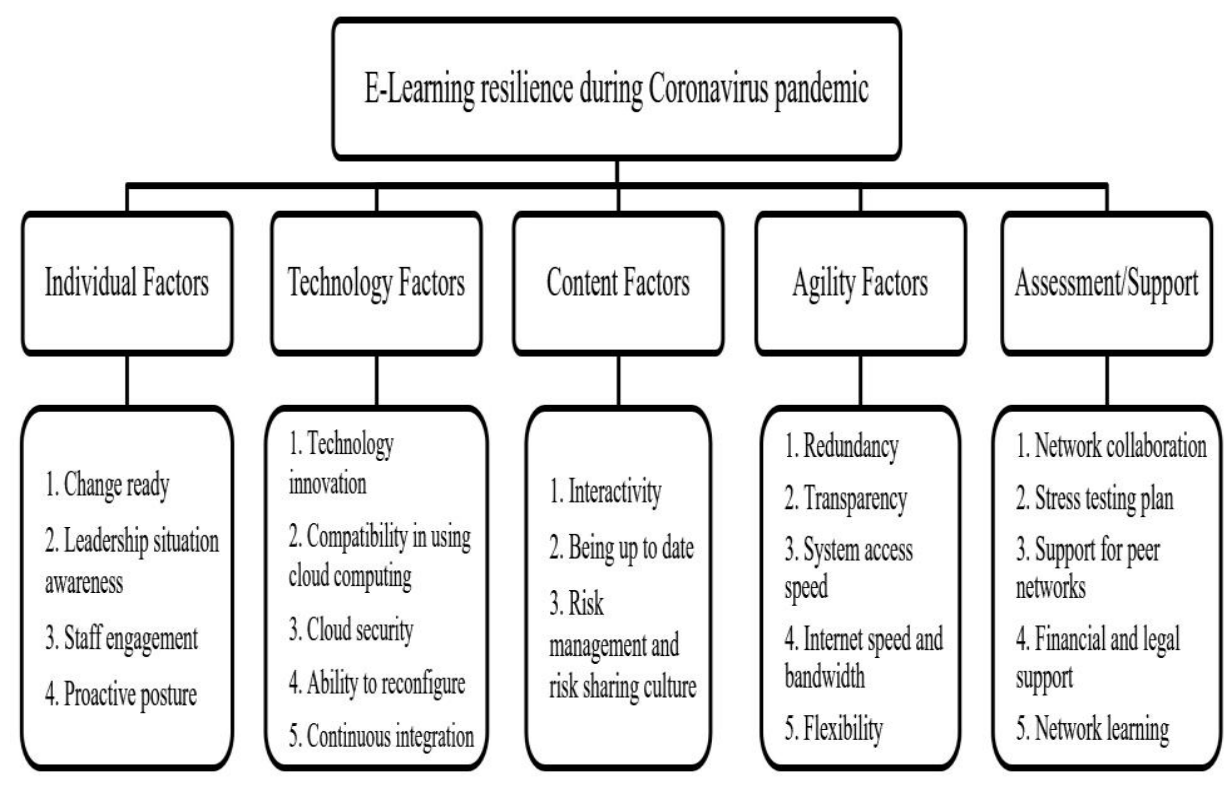

Fig. 2: E-learning system resilience assessment using the ANFIS model.

\subsection{ANFIS model}

To assess the validity of the ANFIS model, it is developed along with a questionnaire consisting of open and closed questions to a number of experts in the field of resilience of e-learning systems; their approvals were evaluated and their points of view were obtained step by step. Then, necessary modifications to the conceptual model of the research were made. The process of making changes continued until reaching convergence among experts. According to the results of the final perspectives and the use of statistical techniques, the validity of the conceptual model was investigated.

According to Fig. 3, the main ANFIS is designed to evaluate the resilience of elearning. The input layer consists of 22 features of five main factors including individual, technology, content, and agility, and assessment/support factors (as summarized in Fig. 2). The resilience capability of the e-learning is considered as the output of the ANFIS model. The hidden layer includes fuzzy operator, application method, aggregation of all outputs, and defuzzification. 


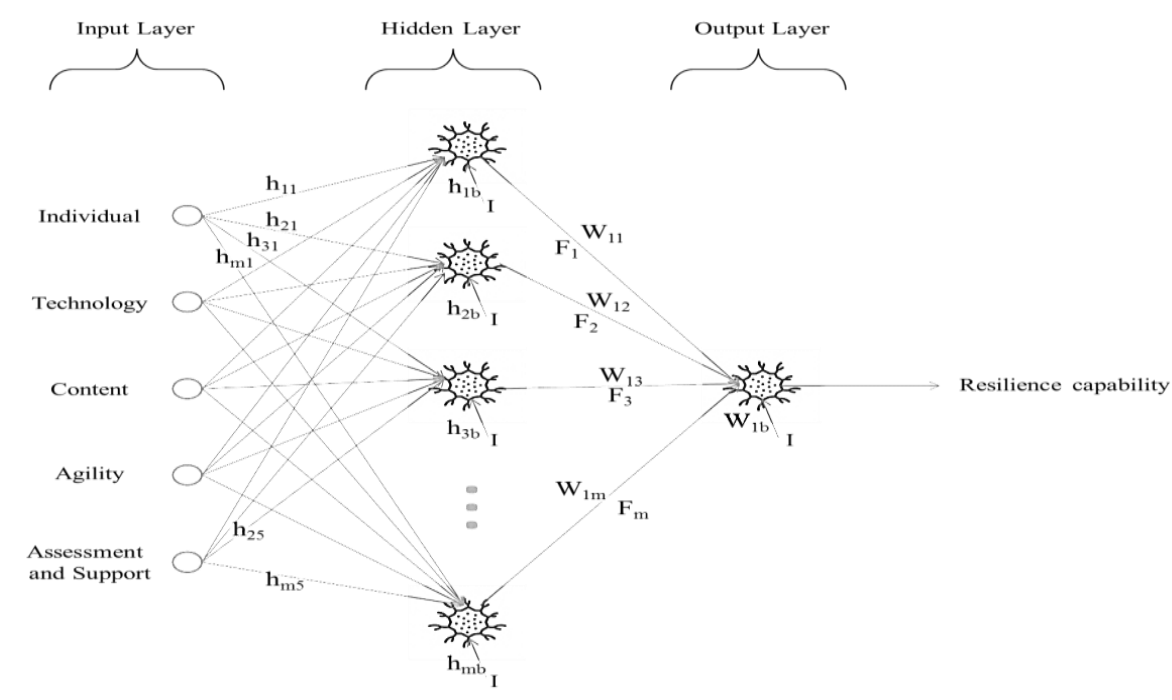

Fig. 3: ANFIS architecture to evaluate the resilience of e-learning.

\subsection{Performance measures}

After training of the ANFIS model on training dataset, the quality of the model to evaluate the e-learning resilience can be determined using Root Mean Square Error (RMSE), Mean Absolute Error (MAE), and correlation (R) between the predicted resilience values and those of actual resilience values given by the expert, which can be expressed as

$$
\begin{gathered}
R M S E=\sqrt{\frac{1}{N} \sum_{j=1}^{N}\left(\text { pred }_{j}-\text { act }_{j}\right)^{2}} \\
M A E=\frac{1}{N} \sum_{j=1}^{N} \mid \text { pred }_{j}-a c t_{j} \mid \\
R=\frac{\frac{1}{N} \sum_{j=1}^{N} \mid \text { pred }_{j}-\overline{\text { pred }}|\times| a c t_{j}-\overline{\text { act }} \mid}{\sqrt{\frac{1}{N} \sum_{j=1}^{N}\left(\text { pred }_{j}-\overline{\text { pred }^{2}}\right)^{2}} \times \sqrt{\frac{1}{N} \sum_{j=1}^{N}\left(\text { act }_{j}-\overline{\text { act }}\right)^{2}}}
\end{gathered}
$$

where predj is the predicted resilience and act $\mathrm{j}$ is the actual resilience for $\mathrm{j}$-th sample. Moreover, $\mathrm{N}$ is the total number of test data samples.

\section{Model Implementation}

\subsection{Settings}

In order to evaluate the resilience of the e-learning system, the presented ANFIS 
model has been implemented on the virtual Iranian university, including 23 units of virtual universities with undergraduate and graduate students. Following the outbreak of COVID-19, all of the educational units in Iran were closed from February 2020, based on the Iranian government instructions. Despite the recent closures, two basic measures have been taken to protect the educational process: (a) developing "SHAD" application to primary, secondary, and high schools as well as (b) launching educational systems by universities across the country. Allocating a certain portion of Internet traffic for free to students, teachers, and professors and developing passive defense infrastructure, etc., remain two taken measures of the resilient proceedings during the outbreak of COVID-19 in Iran.

In this paper, Gaussian functions have been employed to define input and output variables of the ANFIS model, as seen in Fig. 4. The range of changes in input and output variables is defined between 0 and 10. By structuring fuzzy inference rules to extract experts' knowledge, a questionnaire containing a combination of different valuesfor input variables was prepared. Different valuesare generated randomly and experts are asked to judge the input and output variables based on practical knowledge and experience. In this paper, subtractive clustering has been utilized to form the structure of inferential rules to estimate the number of categories and data centers in the dataset. The influence factor, acceptance rate, and rejection rate have values of $0.15,1.25$, and 0.5 , respectively, according to the subtractive clustering process.

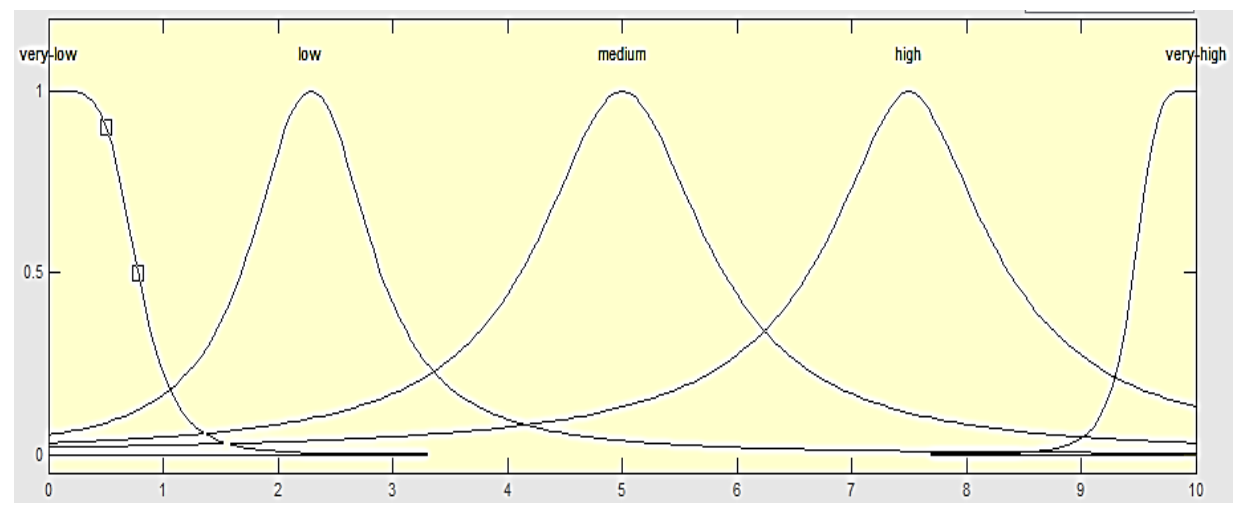

Fig. 4: The membership function for the linguistic variables.

\subsection{Results of ANFIS model}

To design the proposed ANFIS model, an optimal training method was used. Error Tolerance (ET) was employed to determine a criterion for stopping training directly related to the MAE. The designed ANFIS with 70 epochs achieved an acceptable rate of error. Table 2 shows the error rates in ANFIS and Sub-ANFIS parameters. 
Table 2: Error rates in different ANFIS parameters

\begin{tabular}{|c|c|}
\hline ANFIS Stage & Error \\
\hline Individual (IF) & $1.37 * 10-2$ \\
\hline Technology (TF) & $1.4 * 10-2$ \\
\hline Content (CF) & $6.62 * 10-2$ \\
\hline Agility (AF) & $3.83 * 10-2$ \\
\hline Assessment and support (SF) & $5.62 * 10-2$ \\
\hline Resilience of e-learning (RE) & $1.93 * 10-2$ \\
\hline
\end{tabular}

The defuzzified crisp value of resilience has been achieved as $R E=3.41$; by matching this value with the defined membership functions, the initial resilience of the e-learning system of the virtual Iranian university can be assessed by almost medium to low degrees of resilience. In order to validate the designed model, the information extracted from experts' knowledge and judgment on the output variable of ANFIS (under different values of its inputs) is divided into three categories: training data, testing data, and checking data. To train the ANFIS model, a hybrid training technique (combination of descending gradient and least error squares) has been used. In this regard, error tolerance is used to set an objective, that is, to stop training when it directly corresponds to the size of the error. Training stops when training data error is within the defined error range.

According to the subtractive clustering process, the influence factor, squash factor, acceptance ratio, and reject ratio are characterized by values of $0.5,1.25,0.5$, and 0.15 , respectively. The hybrid method has been used for ANFIS training. Error tolerance is employed to determine a criterion for stopping training that is directly related to the size of the error. The trend of errors associated with the designed ANFIS was investigated; for example, Fig. 5 shows the trend of reducing the training error in return for increasing epochs. Figure 6 clearly demonstrates the consistency between the training and checking data sets. In this diagram, asterisk and plus signs denote the output of the system and the checking data, respectively, which are almost consistent, thus demonstrating the absence of over-compliance in the designed ANFIS. 


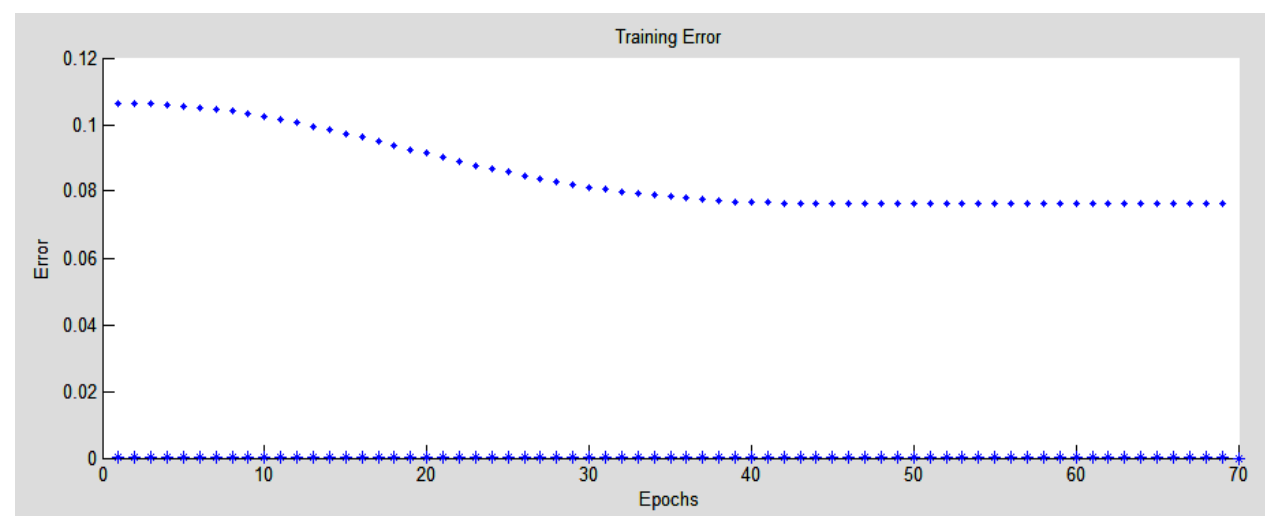

Fig. 5: Trend of error changes in ANFIS resilience capabilities.

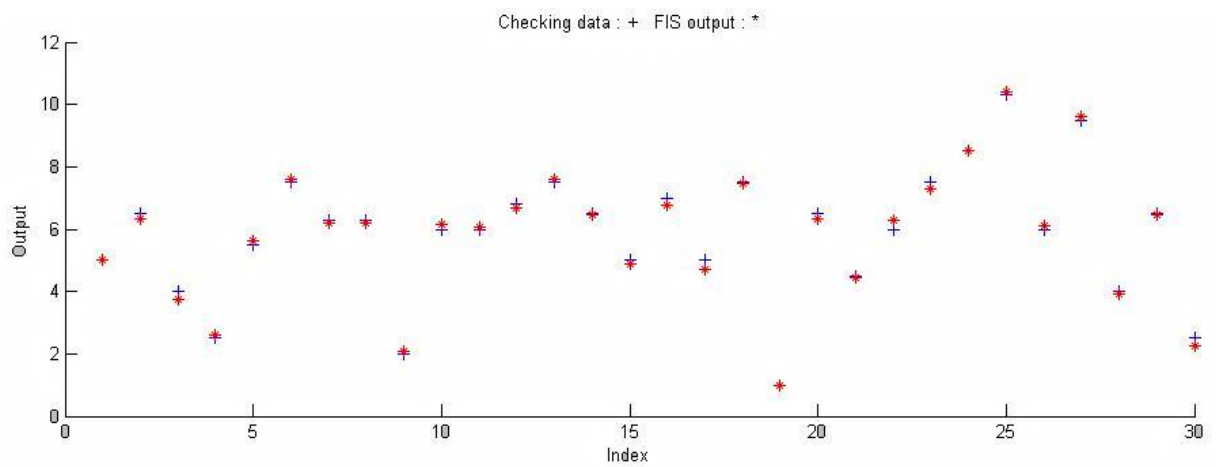

Fig. 6: Comparison chart between ANFIS output and checking data.

Comparison of the ANFIS test data designed to evaluate resilience enablers can be seen in Fig. 7. The asterisk symbol indicates the ANFIS output and the circle symbol shows the testing data. In this diagram, the average error calculated in this ANFIS is 0.056 .

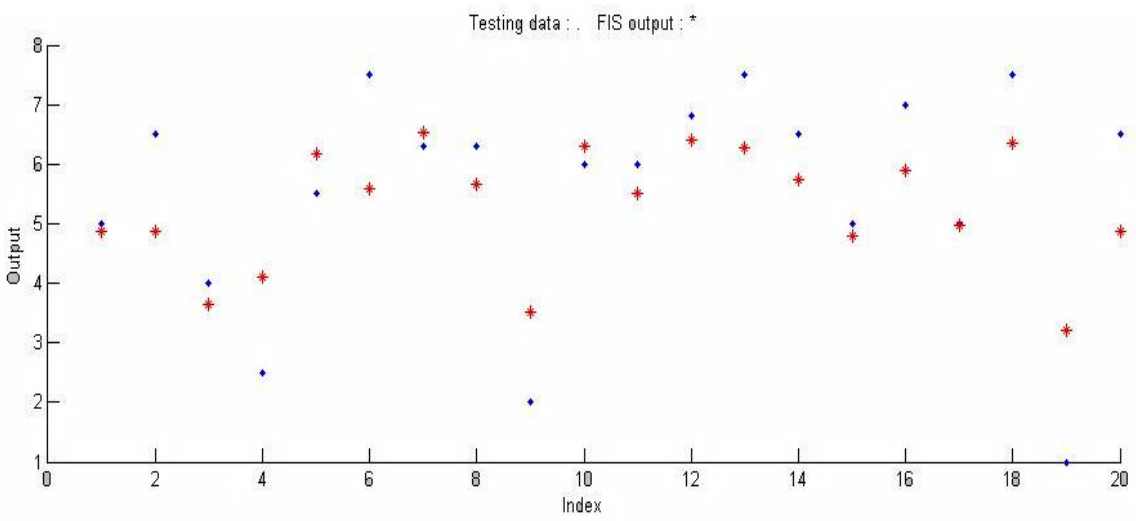

Fig. 7: Comparison between ANFIS output and testing data. 


\subsection{Comparision with other machine learning models}

To justify the performance of the proposed ANFIS model to evaluate the resilience of e-learning, it is compared with multilayer perceptron (MLP), K-nearest neighborhood (KNN), decision trees (DT), support vector machine (SVM), and naïve bayes (NB). Comparison of the obtained results by different machine learning models are provided in Table 3. According to the obtained results, the proposed ANFIS model is superior against the compared models in term of minimizing the error rate in evaluating the e-learning resilience.

Table 3: Comparision of different techniques, in terms of MAE, RMSE, and R.

\begin{tabular}{|c|c|c|c|}
\hline Algorithm & RMSE & MAE & R \\
\hline MLP & 0.863 & 0.941 & 0.859 \\
\hline KNN (K=3) & 0.907 & 0.963 & 0.852 \\
\hline DT & 0.841 & 0.935 & 0.883 \\
\hline SVM (RBF) & 0.812 & 0.899 & 0.89 \\
\hline NB & 0.89 & 0.955 & 0.861 \\
\hline $\begin{array}{c}\text { ANFIS } \\
\text { (Proposed) }\end{array}$ & 0.783 & 0.852 & 0.906 \\
\hline
\end{tabular}

\subsection{Findings and discussion}

Statistical tests were carried out to evaluate the verification of the fuzzy inference system. In this method, experts in the field of e-learning were asked to express their views on the output of the system in terms of different, randomly generated input values. Then, the existing views were compared with the actual outputs of the designed system. Considering $\alpha=0.05$ and using SPSS software, the P-value was calculated to be 0.117 , which was greater than $\alpha$. As a result, the hypothesis of equality of means is not rejected, which means that there is no significant difference between the designed ANFIS output and the knowledge of experts.

Assuming $\mathrm{IF}=6, \mathrm{TF}=7, \mathrm{CF}=5, \mathrm{AF}=4$, and $\mathrm{SF}=2$, the resilience rate in the case study was achieved as $\mathrm{RE}=3.41$. By changing one input and keeping the other four inputs unchanged each time and for each input, Table 4 provides the sensitivity of the model in exchange for a change in each of the inputs. The first row indicates the current situation and the following rows show addition of one unit to each of the factors and the amount of resilience calculated. According to the obtained results in Table 4, the agility factor has caused a significant change of 4.71 in resilience such that it has transformed resilience from "medium-low" to "medium". Sensitivity analysis shows that after the agility factor, technology and assessment/support factors can be considered as the next priorities in virtual Iranian university. 
Table 4: Sensitivity analysis of the model.

\begin{tabular}{|c|c|c|c|c|c|c|}
\hline Values & IF & TF & CF & AF & SF & RE \\
\hline Default Values & 6 & 7 & 5 & 4 & 2 & 3.41 \\
\hline Changing in IF & 7 & 7 & 5 & 4 & 2 & 3.53 \\
\hline Changing in TF & 6 & 8 & 5 & 4 & 2 & 4.01 \\
\hline Changing in CF & 6 & 7 & 6 & 4 & 2 & 3.54 \\
\hline Changing in AF & 6 & 7 & 5 & 5 & 2 & 4.71 \\
\hline Changing in SF & 6 & 7 & 5 & 4 & 3 & 3.81 \\
\hline
\end{tabular}

The concept of redundancy along with flexibility, transparency, ease of access to software and hardware infrastructure, and appropriate internet bandwidth are appropriate indicators identified for agility and they can ensure high resilience for elearning systems. E-learning has the potential to reach the public in critical situations such as COVID-19 epidemic and this objective is realized upon achieving agility and technological development. The combination of redundancy and flexibility can prevent negative effects and reduce the costs of redundancy through greater transparency. It can be expected that given the new needs of today's human beings such as rapid access to computer, intelligent phones, and the internet with appropriate speed as well as bandwidth and other potentials, the effort to implement and use the benefits of e-learning should be more than ever.

\section{Conclusion}

Given the importance of the resilience of any system during the COVID-19 outbreak, the corresponding factors should be properly addressed so that the degree of e-learning resilience can be determined. In this regard, five significant factors have been identified, including individual, technology, content, agility, and evaluation/support. Because of the qualitative nature of resilience, employing a powerful tool such as the developed ANFIS model for the resilience assessment can be propounded. To prove the applicability of the proposed model, it has been applied to virtual Iranian university, and the implemented model for them has a "medium-low" resilience. The proposed model has shown significant sensitivity to changes in the agility factor. Therefore, agility is considered as the first priority in achieving the desired level of resilience in the e-learning system of virtual Iranian university. This model can be used in all educational institutions to assess the resilience of e-learning systems. To implement the presented model for different organizations, the values of designed ANFIS model should be defined specifically for every organization and the corresponding model need to be simulated by 
examining the involved components and relationships. For further research, other fuzzy-based neural networks such as Co-Active Neuro-Fuzzy Inference System (CANFIS) can be considered in investigating the multiplicity of outputs for the proposed problem.

\section{References}

Al-Balas, M., Al-Balas, H. I., Jaber, H. M., Obeidat, K., Al-Balas, H. I. \& Aborajooh, E. A. (2020). Distance learning in clinical medical education amid COVID-19 pandemic in Jordan: current situation, challenges, and perspectives, BMC Med Educ., 20(1), 341.

Alhusban, A. A., Alhusban, S. A., \& Alhusban, M. A. (2021). How the COVID 19 pandemic would change the future of architectural design. Journal of Engineering, Design and Technology.

Aljawarneh, S. A. (2019). Reviewing and exploring innovative ubiquitous learning tools in higher education. Journal of computing in higher education, 32(1), 57-73.

Almulhim, A. Y., Almulhim, S. A., Almulhim, A. A. \& Khan, A. S. (2020) The impact of e-learning modalities on medical students in KSA during the COVID-19 pandemic. Journal of Taibah University Medical Sciences, 15 (5), 437-438.

Ayebi-Arthur, K. (2017). E-learning, resilience and change in higher education: Helping a university cope after a natural disaster, E-Learning and Digital Media, 14(5), 259-274.

Bonomi, S., Sarti, D. \& Torre, T. (2020). Creating a collaborative network for welfare services in public sector. A knowledge-based perspective, Journal of Business Research, 112(1), 440-449.

De Meo, P., Messina, F., Rosaci, D. \& Same, G. M. L. (2017). Combining Trust and Skills Evaluation to Form E-Learning Classes in On-line Social Networks. Information Sciences, 405, 107-122.

Dignan, L. (2020). Online learning gets its moment due to COVID-19 pandemic: Here's how education will change. https://www.zdnet.com/article/online-learninggets-its-moment-due-to-covid-19-pandemic-heres-how-education-will-change.

Favale, T., Soro, F., Trevisan, M., Drago, I. \& Mellia, M. (2020). Campus traffic and e-Learning during COVID-19 pandemic. Computer Networks, 176, 107290. 
Francis, R. \& Bekera, B. (2014). A metric and frameworks for resilience analysis of engineered and infrastructure systems. Reliab. Eng. Syst. Safety, 121(1), 90-103.

Jang, J. S. R. (1993). ANFIS: adaptive network-based fuzzy inference systems, IEEE Trans Sys Man Cybern, 23, 665-685.

Li, Y. \& Zobel, C. (2020). Exploring supply chain network resilience in the presence of the ripple effect, International Journal of Production Economics, 228(1), 107693.

Lipscomb, S. T., Hatfield, B., Lewis, H., Goka-Dubose, E. \& Fisher, P. A. (2019). Strengthening children's roots of resilience: Trauma-responsive early learning. Children and Youth Services Review, 107, 104510.

Moreira, F., Pereira, C. S., Durão, N. \& Ferreira, M. J. (2018). A comparative study about mobile learning in Iberian Peninsula Universities: Are professors ready? Telematics and Informatics, 35, 979-992.

Nabizadeh, M., Khalilzadeh, M., Ebrahimnejad, S., \& Ershadi, M. J. (2021). Developing a fuzzy goal programming model for health, safety and environment risks based on hybrid fuzzy FMEA-VIKOR method. Journal of Engineering, Design and Technology.

Naqshbandi, M. M., Ainin, S., Jaafar, N. I., \& Shuib, N. L. M. (2017). To Facebook or to Face Book? An investigation of how academic performance of different personalities is affected through the intervention of Facebook usage. Computers in Human Behavior, 75, 167-176.

Oliveira, A., Camarinha-Matos, L. \& Inês Ferrada, F. (2015). Supporting Collaborative Networks for Complex Service-Enhanced Products. Working Conference on Virtual Enterprises PRO-VE 2015: Risks and Resilience of Collaborative Networks, 181-192.

Pilevari, N., Rahmaty, M., \& Shokouhifar, M. (2021). Combined ANFIS-Dijkstra's Model: A Dynamic Simulation for Suburban Public Transportation. Journal of System and Management Sciences, 11(1), 155-175.

Shokouhifar, M., \& Jalali, A. (2017). Optimized sugeno fuzzy clustering algorithm for wireless sensor networks. Engineering applications of artificial intelligence, 60, $16-25$. 
Shokouhifar, M., Sabbaghi, M. M., \& Pilevari, N. (2021). Inventory management in blood supply chain considering fuzzy supply/demand uncertainties and lateral transshipment. Transfusion and Apheresis Science, 103103.

Shokouhifar, M. (2021a). FH-ACO: Fuzzy heuristic-based ant colony optimization for joint virtual network function placement and routing. Applied Soft Computing, $107,107401$.

Shokouhifar, M. (2021b). Swarm Intelligence RFID Network Planning using MultiAntenna Readers for Asset Tracking in Hospital Environments. Computer Networks, 108427.

Simpeh, F., \& Amoah, C. (2021). COVID-19 guidelines incorporated in the health and safety management policies of construction firms. Journal of Engineering, Design and Technology.

The, M. M. \& Usagawa, T. (2018). Change in E-learning Readiness and Challenge for Myanmar Higher Education. Creative Education, 9, 1277-1286.

UNESCO (2020). Why the world must urgently strengthen learning and protect finance for education. https://en.unesco.org/news/why-world-must-urgentlystrengthen-learning-and-protect-finance-education.

Van de Laar, M. (2020). E-resilience in education: A conceptual framework. United Nations University, ISBN 978-92-808-5017-8. 